\title{
Análisis de la percepción del estudiante sobre el empleo didáctico de Scratch en el aula
}

Esteban Vázquez-Cano ${ }^{a}$ y Paz Díez-Arcón ${ }^{b}$

a'Universidad Nacional de Educación a Distancia (UNED): evazquez@edu.uned.es y buniversidad Nacional de Educación a Distancia (UNED): pazdiez@pas.uned.es

\section{\$EWWDFW}

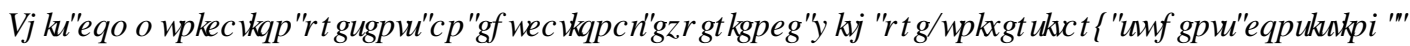

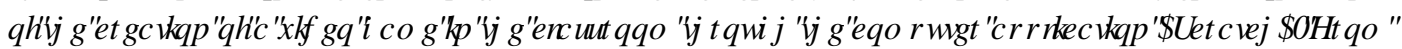

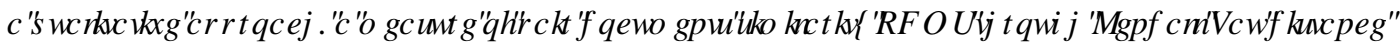

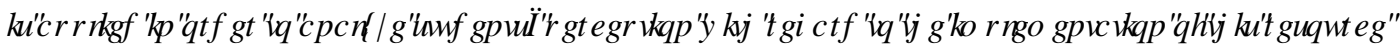

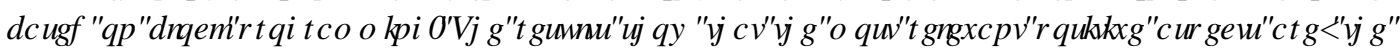

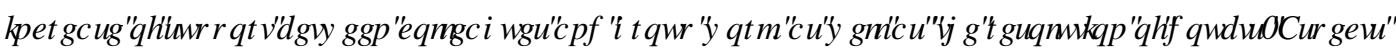

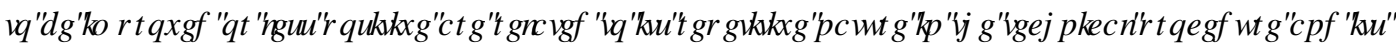

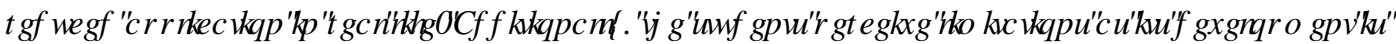

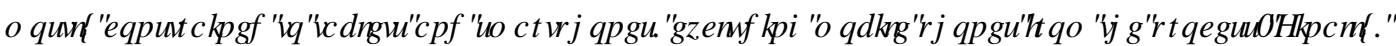

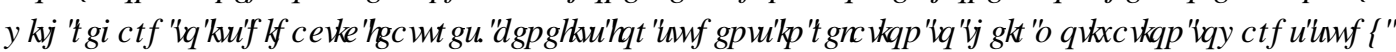

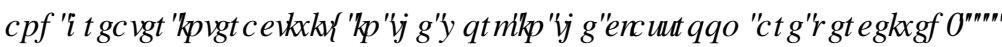

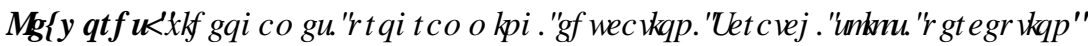

]

\section{HXP HQ}

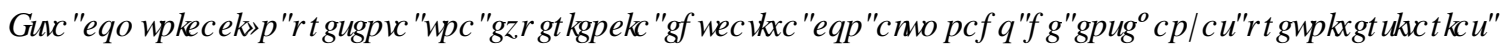

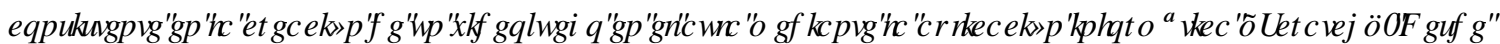

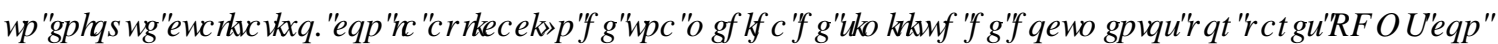

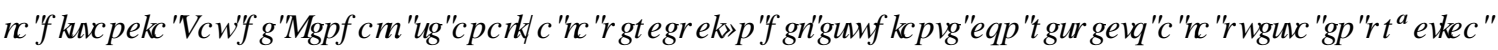

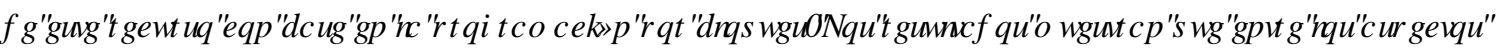

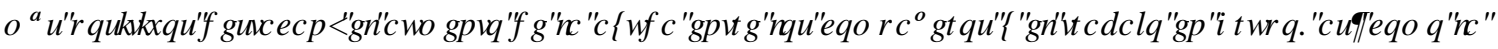

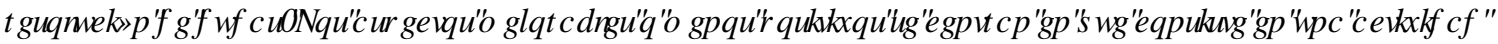

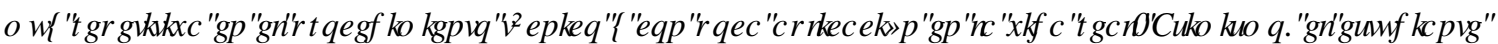

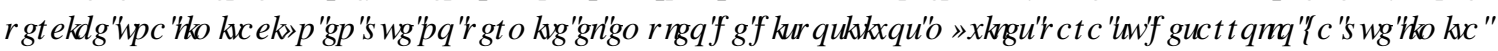

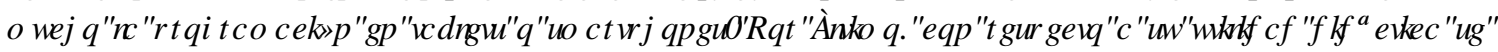

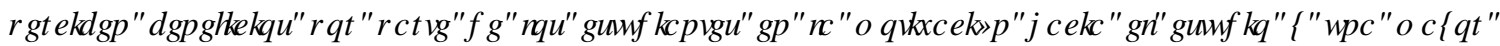
IQULFWMIGDGHQHOWDEDNHQHODXQD [

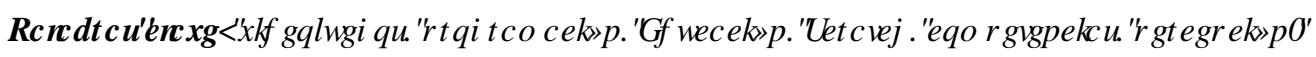

\section{Introducción}

La mayoría de los proyectos de videojuegos en el aula se basan en su uso didáctico principalmente desde principios didácticos inspirados en la gamificación(Gamification) o en el aprendizaje basado en juegos (Game-Based Learning) (Zichermann, \& Cunningham, 2011; Deterding, 2012). Estas iniciativas incorporan al aula el videojuego como un recurso didáctico en apoyo de diferentes asignaturas y contenidos, pero recientemente se están incorporando nuevos proyectos basados en el movimiento global liderado 


\section{\$ Q OMVGHOSSHFHFYQIGHOHXGDQMVEUHHOP SGRGG FUFRGH6FWWKIHQHDDXO]}

principalmente por Code.org y CodeAcademy que promueven la enseñanza de la programación informática en las escuelas, poniendo el foco en las excelentes posibilidades didácticas y laborales que se abren para los jóvenes que sepan programar (Code.org, 2013; Nicks, 2014). En este estudio presentamos y analizamos una experiencia de creación de videojuegos en el aula de Bachillerato desde una doble perspectiva: didáctica y técnica.

\section{Objetivos}

El siguiente estudio tiene como pretensión reflejar una experiencia educativa llevada a cabo por alumnos preuniversitarios mediante la creación de un videojuego a través de la aplicación "Scratch". También analizar las perspectivas didácticas y técnicas tras la experiencia. Mediante este análisis se objetiva identificar los aspectos más positivos de la utilización de la aplicación, así como los negativos y mejorables. Por otra parte, se pretende obtener la valoración de los aspectos que tienen una influencia en la mejora del aprendizaje o su utilidad didáctica.

\section{Marco teórico}

\subsection{La programación en el aula. Una tendencia mundial}

Enseñar de manera creativa significa adoptar enfoques imaginativos para hacer el aprendizaje más interesante, emocionante y efectivo (Wilson et al., 2013; Sáez et al., 2016; Vázquez-Cano \& Ferrer Delgado, 2015). El uso de los videojuegos ofrece experiencias que promueven satisfacciones intrínsecas y ofrecen oportunidades para el aprendizaje auténtico (Scaffidi \& Chambers, 2012). Existe una tendencia mundial que considera la programación en el aula como una actividad de presente y futuro para el desarrollo de competencias relacionadas con la realidad del mundo laboral y personal de los estudiantes. De esta manera, países como Francia ya desde el curso escolar 2014/15 empezaron a programar en la Educación Primaria (LeJDD, 2014), al igual que Reino Unido tanto para la Educación Primaria como para la Educación Secundaria. Precisamente, en Reino Unido se está viviendo una "revolución tecnológica" en las escuelas y entidades privadas están impulsando "Code camps" (campamentos y cursos de programación para adolescentes) (Jones, 2014), o subvencionando la compra de placas Arduino y Raspberry Pi para que los docentes no se vean frenados por la falta de recursos y puedan formar a los escolares en ciencias de la computación. Otros países como Estonia, llevan ya tiempo enseñando programación a los alumnos de primaria (BBC, 2014), Australia lo hará próximamente y países como Finlandia, Israel, Corea del Sur, Nueva Zelanda o Grecia están desarrollando programas piloto.

En Estados Unidos, figuras clave de la industria tecnológica como Bill Gates, Mark Zuckerberg o Jack Dorsey apoyan el proyecto Code.org (http://code.org/) que busca, precisamente, concienciar a alumnos y profesores en las ventajas de la enseñanza de la programación en las escuelas. En España, y especialmente, en Castilla-La Mancha, inspirados por el movimiento global de la Hora del Código (+RXURI \&RGH que busca enseñar a programar a partir de los cuatro años). Se considera que al introducir al estudiante tempranamente en las ciencias computacionales, tendrán una base para el éxito en cualquier carrera del siglo XXI (http://studio.code.org/). El uso de la programación en las aulas va más allá de una necesidad social o de proyección profesional de los estudiantes y estudios recientes han demostrado que aprender a programar tiene un impacto positivo en la creatividad y respuesta emocional de niños con dificultades de aprendizaje (Gold, 2011), así como en el desarrollo de las habilidades cognitivas y socio-emocionales (Kordaki, 2012; Fessakis et al., 2013).

Asimismo, el uso de herramientas digitales y entornos virtuales convierten el proceso de enseñanzaaprendizaje en una actividad más social y colaborativa al promover una mayor interacción para su 
desarrollo. Investigaciones en el inicio del siglo XXI ya demostraban que cuando los niños se encuentran trabajando con un ordenador hablan usando más del doble de palabras por minuto que cuando se encuentran realizando otras actividades sin utilizar dispositivos electrónicos (Wartella \& Jennings, 2000). Además, cuando los niños están trabajando con el ordenador es más probable que busquen la asistencia y los consejos de otros compañeros, incluso si hay un adulto presente, incrementando la socialización entre los compañeros (LTS, 2010). Asimismo, cuando se cuenta con un ordenador por estudiante, los niños tienden a formar grupos mientras trabajan con dispositivos tecnológicos (Druin, 1998). Por último, existen evidencias científicas que demuestran que el alumnado que aprende a programar en edades tempranas tiene menos estereotipos de género en relación a las carreras científicas: Ciencias, Tecnología, Ingeniería y Matemáticas (Burke \& Mattis, 2007); y menos reticencias para continuar sus estudios y profesiones en estas disciplinas (Matlin, 1993).

\section{Desarrollo de la innovación}

\subsection{Descripción de la experiencia didáctica con Scratch}

La experiencia didáctica fue creada por el profesor Desiderio Ferrer Delgado en el IES Margarita Salas de Seseña y se desarrolló a través de un aula virtual creada con la herramienta de software libre eXelearning en la que se alberga una clase virtual con apuntes, videos, fotos y recursos de descarga. Los recursos creados en eXelearning son accesibles en formato XHTML o HTML5, pudiendo generarse sitios web completos (páginas web navegables), insertar contenidos interactivos (preguntas y actividades de diferentes tipos) en cada página, exportar los contenidos creados en otros formatos como ePub3 (un estándar abierto para libros electrónicos), IMS o SCORM (estándares educativos que permiten incorporar los contenidos en herramientas como Moodle), XLIFF (un estándar para la traducción) y catalogar los contenidos con diferentes modelos de metadatos: Dublin Core, LOM, LOM-ES.

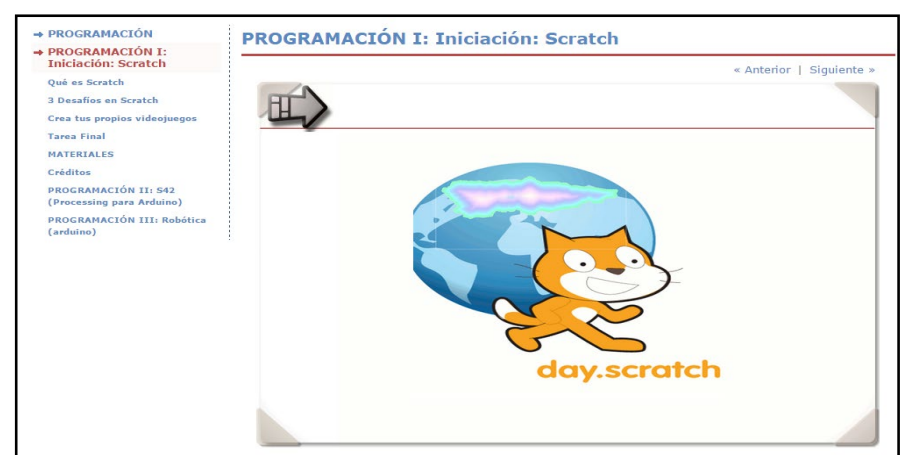

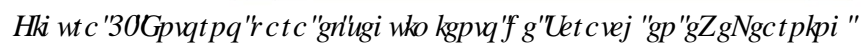

La estructura pedagógica de la actividad se programó durante la tercera evaluación atendiendo a la siguiente secuencia pedagógica:

\section{Objetivos curriculares:}

1. Familiarizarse con los elementos básicos de la interfaz hombre-máquina.

2. Conocer los fundamentos físicos y lógicos de los sistemas ligados a estas tecnologías.

3. Manejar las estrategias que permiten convertir estas tecnologías en instrumentos de diseño, simulación, fabricación y control.

4. Emplear técnicas de búsqueda, elaboración y presentación de la información con criterios de realidad científica.

5. Utilizar las herramientas propias de estas tecnologías para adquirir, analizar y transformar la información, convirtiéndola en fuente de conocimiento. 


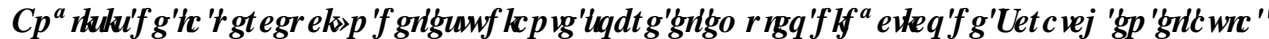

6. Diseñar, actualizar y consultar la información de bases de datos relacionales.

7. Utilizar lenguajes de programación para la resolución de problemas de diferentes ámbitos, entre los que se incluyen proyectos sencillos de control.

8. Aplicar herramientas de análisis y diseño asistido por ordenador a la elaboración de un producto.

9. Discriminar qué instrumento es más adecuado para un determinado problema científico o creativo.

10. Usar los recursos informáticos como instrumento de resolución de problemas específicos.

\section{Competencias:}

a. Competencia en comunicación lingüística

b. Competencia matemática.

c. Competencia en el conocimiento y la interacción con el mundo físico.

d. Tratamiento de la información y competencia digital

e. Competencia social y ciudadana.

f. Competencia cultural y artística.

g. Competencia para aprender a aprender

h. Autonomía e iniciativa personal

\section{Indicadores de desarrollo:}

1. Conoce los elementos básicos de la aplicación Scratch.

2. Utiliza adecuadamente las herramientas de la aplicación.

3. Analiza e identifica los elementos programáticos.

4. Selecciona y utiliza los elementos de control y las funciones básicas de programación.

5. Crea en equipo un videojuego

6. Evalúa el videojuego creado por otro equipo.

Se realizaron procesos de autoevaluación, coevaluación y evaluación final de cada uno de los desafíos y de la tarea final realizada por cada grupo de estudiantes. Para orientar el procedimiento de evaluación, se diseñó una rúbrica que fue utilizada tanto por los alumnos en grupo para la coevaluación, como por el profesor para la evaluación del proyecto final (Tabla 1).

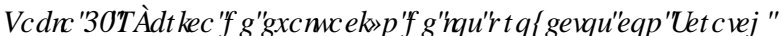

\begin{tabular}{|c|c|c|c|c|c|}
\hline Criterios & $\begin{array}{c}2 \\
\text { (negativa) }\end{array}$ & $\begin{array}{c}4 \\
\text { (incorrecta) }\end{array}$ & $\begin{array}{c}5 \\
\text { (correcta) }\end{array}$ & $\begin{array}{c}7 \\
\text { (perfecta) }\end{array}$ & $\begin{array}{c}9 \\
\text { (excelente) }\end{array}$ \\
\hline $\begin{array}{c}\text { Pantalla de } \\
\text { presentación } \\
(5 \%)\end{array}$ & $\begin{array}{l}\text { No tiene pantalla } \\
\text { de presentación }\end{array}$ & $\begin{array}{l}\text { El programa no } \\
\text { inserta diálogos que } \\
\text { informen al usuario } \\
\text { sobre el objetivo de } \\
\text { la aplicación y su } \\
\text { funcionamiento. }\end{array}$ & $\begin{array}{l}\text { El programa presenta } \\
\text { una pantalla sin } \\
\text { animación con el } \\
\text { manejo básico del } \\
\text { programa. }\end{array}$ & $\begin{array}{l}\text { El programa presenta } \\
\text { una pantalla animada } \\
\text { en la cual informa al } \\
\text { usuario sobre el } \\
\text { objetivo, las reglas y el } \\
\text { manejo del juego en un } \\
\text { formato atractivo. }\end{array}$ & $\begin{array}{l}\text { Presenta una pantalla } \\
\text { animada con el } \\
\text { nombre del juego. } \\
\text { Existe un botón para } \\
\text { ver las instrucciones, } \\
\text { y otro para empezar a } \\
\text { jugar. }\end{array}$ \\
\hline $\begin{array}{c}\text { Funcionamiento } \\
\qquad(30 \%)\end{array}$ & $\begin{array}{l}\text { El juego apenas } \\
\text { funciona, tiene un } \\
\text { único nivel, no } \\
\text { lleva cuenta de } \\
\text { vidas ni puntos ni } \\
\text { tiempo, no tiene } \\
\text { pantallas de fin. }\end{array}$ & $\begin{array}{l}\text { El juego solo } \\
\text { presenta un nivel y } \\
\text { no lleva cuenta de } \\
\text { vidas o puntos. No } \\
\text { presenta pantallas } \\
\text { de Game Over ni } \\
\text { The End }\end{array}$ & $\begin{array}{l}\text { El juego presenta } \\
\text { algún fallo, no tiene } \\
\text { muchos niveles y el } \\
\text { marcador o la cuenta } \\
\text { de vidas no } \\
\text { funcionan bien. } \\
\text { Presenta pantallas de } \\
\text { Game Over y The } \\
\text { End }\end{array}$ & $\begin{array}{l}\text { Funciona } \\
\text { perfectamente, tiene } \\
\text { varios niveles } \\
\text { atractivos y } \text { bien } \\
\text { diseñados y lleva } \\
\text { marcador y vidas. Hay } \\
\text { pantalla de Game over } \\
\text { y The End. }\end{array}$ & $\begin{array}{l}\text { Funciona } \\
\text { perfectamente, tiene } \\
\text { varios niveles } \\
\text { atractivos y bien } \\
\text { diseñados y lleva } \\
\text { marcador y vidas. } \\
\text { Hay pantalla de } \\
\text { Game over y The } \\
\text { End animada. }\end{array}$ \\
\hline $\begin{array}{c}\text { Programación } \\
(30 \%)\end{array}$ & $\begin{array}{l}\text { Apenas existe } \\
\text { programación. }\end{array}$ & $\begin{array}{l}\text { El juego tiene una } \\
\text { programación muy } \\
\text { básica que no } \\
\text { demuestra gran } \\
\text { conocimiento de las }\end{array}$ & $\begin{array}{l}\text { El juego muestra } \\
\text { cierto entendimiento } \\
\text { de las estructuras de } \\
\text { control de } \\
\text { programación, pero }\end{array}$ & $\begin{array}{l}\text { Se han optimizado casi } \\
\text { todas las instrucciones } \\
\text { y no hay repetición de } \\
\text { comandos innecesaria, } \\
\text { y se han usado la }\end{array}$ & $\begin{array}{l}\text { El juego incorpora } \\
\text { para } \\
\text { programación una } \\
\text { gran variedad de } \\
\text { herramientas de }\end{array}$ \\
\hline
\end{tabular}




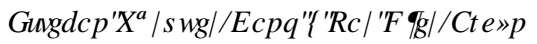

\begin{tabular}{|c|c|c|c|c|c|}
\hline & & $\begin{array}{lr}\text { estructuras } & \text { de } \\
\text { control. } & \text { Las } \\
\text { variables no } & \text { están } \\
\text { inicializadas. } & \end{array}$ & $\begin{array}{l}\text { se puede optimizar } \\
\text { haciendo mejor uso } \\
\text { de las mismas. Existe } \\
\text { inicialización de las } \\
\text { variables, se parte de } \\
\text { un estado conocido. }\end{array}$ & $\begin{array}{ll}\text { mayor parte de } & \text { de } \\
\text { instrucciones } & \text { de } \\
\text { control. Se usan flags. }\end{array}$ & $\begin{array}{l}\text { control, más allá de } \\
\text { lo aplicado en los } \\
\text { desafíos } \\
\text { programación } \\
\text { iniciales. }\end{array}$ \\
\hline $\begin{array}{l}\text { Jugabilidad } \\
(20 \%)\end{array}$ & $\begin{array}{l}\text { Es un juego } \\
\text { conocido } \\
\text { implementado de } \\
\text { forma muy pobre. }\end{array}$ & $\begin{array}{l}\text { Es un juego } \\
\text { conocido pero no } \\
\text { implementa toda la } \\
\text { funcionalidad. }\end{array}$ & $\begin{array}{l}\text { Se trata de un juego } \\
\text { conocido } \\
\text { implementado } \\
\text { perfectamente }\end{array}$ & $\begin{array}{l}\text { El juego se basa en un } \\
\text { juego conocido } \\
\text { incorporando } \\
\text { nuevor }\end{array}$ & $\begin{array}{l}\text { El juego es original, } \\
\text { muy jugable y } \\
\text { adictivo. }\end{array}$ \\
\hline $\begin{array}{c}\text { Gráficos } \\
(15 \%)\end{array}$ & $\begin{array}{l}\text { no hay } \\
\text { animaciones ni } \\
\text { gráficos } \\
\text { originales. No hay } \\
\text { referencias ni se } \\
\text { respetan licencias. }\end{array}$ & $\begin{array}{l}\text { las animaciones son } \\
\text { bruscas y no } \\
\text { originales. }\end{array}$ & $\begin{array}{l}\text { existen algunas } \\
\text { animaciones, pero no } \\
\text { son originales. }\end{array}$ & $\begin{array}{l}\text { Las animaciones son } \\
\text { suaves y abundantes. }\end{array}$ & \begin{tabular}{lrr} 
Se usan & \multicolumn{2}{c}{ gráficos } \\
originales y & las \\
animaciones & son \\
suaves y de & gran \\
calidad. Todas & las \\
licencias & son \\
correctas y & bien \\
referenciadas & \\
\end{tabular} \\
\hline
\end{tabular}

La organización de la tarea final consistió en el diseño y desarrollo de un videojuego en grupo de 4 o 5 estudiantes de forma colaborativa. La actividad se desarrolló conforme a las siguientes fases:

Primera fase: Diseño de la narrativa a realizar y argumento.

Segunda fase: distribución de los roles de cada alumno/a.

Tecera fase: creación del videojuego conforme a las siguientes preguntas:

- ¿Qué queremos?

- ¿Qué objetos necesitamos crear?

- ¿Cómo interactúan entre sí?

- ¿Cuántos fondos necesitamos crear?

- ¿Cómo interactúan entre sí y con los objetos?

Los alumnos/as de la clase de bachillerato realizaron varios videojuegos, entre los más destacados se encuentra el siguiente del que realizamos una descripción argumental y técnica de su creación e invitamos al lector a visualizar y jugar en el siguiente enlace: https://scratch.mit.edu/projects/64144384/

Para iniciar el juego se tiene que pinchar la bandera verde. El muñeco salta cuando se presiona la tecla espacio y empieza la música. El objetivo del videojuego es evitar que te atropelle el taxi y evitar chocar con el resto de obstáculos. Cada vez que se juega se dispone de 3 vidas y se va obteniendo puntuación a medida que se salvan los obstáculos.



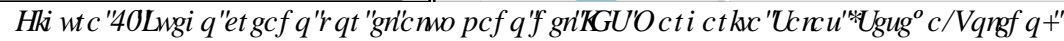

El videojuego se ha diseñado con 11 objetos a los que se ha ido dando movimiento, sonido e interacción en la narrativa de juego con la programación por bloques. 


\subsection{Metodología}

Para pulsar la percepción del estudiante, se les entregó un cuestionario abierto compuesto por tres preguntas: (1) Aspectos más positivos de la experiencia. (2) Aspectos negativos y mejorables (3) Valoración de los aspectos que mejoran en su aprendizaje. Las preguntas 1 y 2 fueron contestadas individualmente y la 3 de forma grupal. La muestra participante estuvo compuesta por 52 estudiantes de primero de bachillerato del IES (32 chicas y 20 chicos) con una media de edad de 16,7 años. Para ello, se construyó una matriz textual por cada una de las tres categorías de preguntas constituida solo para los valores triangulares inferiores y luego se reconstruye para formar la matriz completa. Todos los valores están normalizados entre cero y uno, por lo que se puede tratar como una probabilidad de coincidencia semántica. Usamos una medida de similitud de documentos por pares PDSM (Pairwise Document Similarity Measure) con la distancia Tau de Kendall aplicando la siguiente ecuación:

$$
\operatorname{PDSM}\left(d_{1}, d_{2}, d_{3}\right)=\left(\frac{d_{1 \cap} d_{2 \cap} d_{3}}{d_{1 \cap} d_{2 \cap} d_{3}}\right) \times \frac{P F\left(d_{1}, d_{2}, d_{3}\right)+1}{M-A F\left(d_{1}, d_{2}, d_{3}\right)+1}
$$

La intersección y union de las tres categorías de análisis quedó configurada por tres descriptores: "positivo" "mejorable" "funcionalidad para el aprendizaje" y se calcularon con la siguiente notación ( $\mathrm{w}_{\mathrm{ji}}>0$ es el peso de Len el documento MII

$$
d_{1 \cap} d_{2 \cap} d_{3}=\sum_{i=1}^{M} \operatorname{Min}\left(w_{1 i}, w_{2 i}, w_{3 i}\right)
$$




\section{Resultados}

Los resultados de las comparaciones y similitudes encontradas en las opiniones textuales de los estudiantes se presentan en la matriz de distancias por pares (Figura 3).

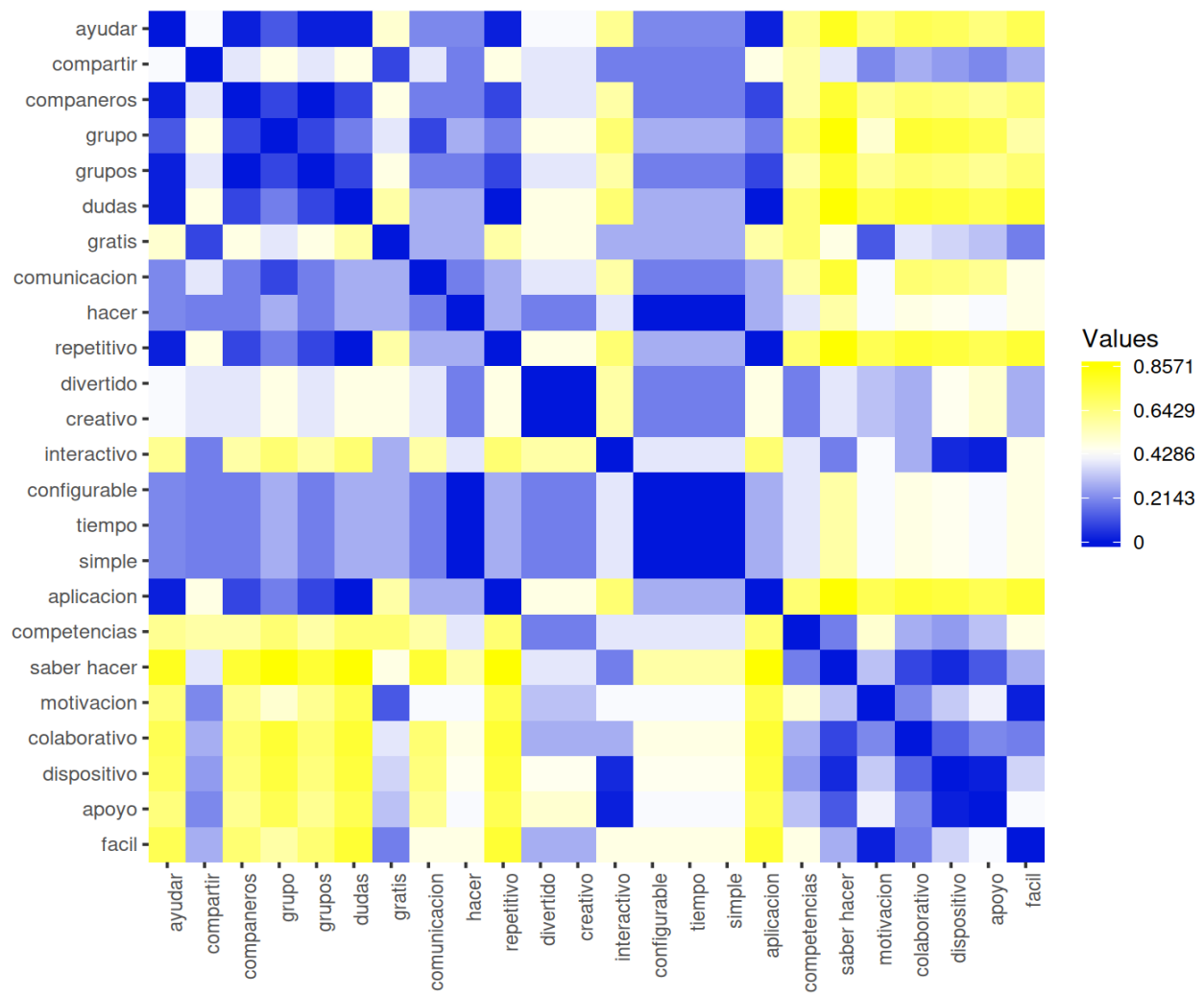



Se puede observar en la Figura 5 que los valores de intermediación más altos entre las 7890 palabras analizadas en las tres categorías analizadas: (1) Aspectos más positivos de la experiencia. (2) Aspectos negativos y mejorables (3) Valoración de los aspectos que mejoran en su aprendizaje, se enfocan en valores superiores a 0.500 en amarillo intenso. Podemos ver que los conceptos con los valores más altos en el conjunto de las tres categorías son: "ayudar" $(0,840)$, "compañeros" $(0,861)$, "grupo/a" $(0,701)$, "dudas" $(0,738)$, entre los aspectos positivos. Entre los mejorables "repetitivo" $(0,835)$, "poca aplicación" $(0,799)$ y "dispositivo" $(0,801)$ dificultad para empleo de tablet y smartphones en su desarrollo. Por último, con respecto a su utilidad didáctica se perciben beneficios por parte de los estudiantes en la "motivación" hacia el estudio $(0,765)$ e "interactivo" $(0,789)$ y facilita el poder en práctica el "saber hacer" $(0.789)$.

\section{Discusión y conclusiones}

El diseño del lenguaje de programación Scratch es un lenguaje visual y no hay que escribir líneas de programación, por tanto, se evitan los errores al teclear; se pueden realizar todo tipo de proyectos y actividades personalizadas. Para conseguir que estos objetivos sean posibles, los creadores de Scratch (Resnick et al. 2009) han introducido tres principios o características básicas en el diseño de este lenguaje de programación. Estos principios son: que la lengua de programación sea lúdica, significativa y social. El lenguaje de programación debe ser lúdico. La idea es que la lengua de programación facilite el juego y que

(c)) BY-NC-ND 2021, Universitat Politècnica de València

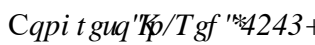




\section{\$ Q OUVGHOSHFHFYQIGHOHXGDQMVEUHHOP SGRGG FUFRIG6 FWWKIHQHODXO]}

se puedan probar, con facilidad, diferentes opciones. Scratch tiene unos "bloques de programación" de diferentes colores, con conectores que permiten que se puedan encajar unos con otros. El objetivo es que los niños puedan jugar con ellos desde el principio y probar a construir sencillos programas (Lamb \& Johnson, 2011). La experiencia al utilizar el lenguaje de programación debe ser significativa. De este modo, en el diseño de Scratch, sus creadores, han dado prioridad a dos criterios del diseño: GYHUGDG (que pueda soportar diferentes tipos de proyectos: historias, juegos, animaciones, simulaciones) y SHURQDD]DFIyQ(que los proyectos se puedan personalizar importando fotos, voces, gráficos, etc.). El uso de la lengua de programación debe propiciar la interacción social. El desarrollo de Scratch va muy unido al desarrollo de su página Web. Para que Scratch tenga éxito necesita que una gran comunidad de personas comparta, apoye, critique, colabore y pueda construir sobre el trabajo de otros. Así el concepto de "compartir" está construido en el entorno de interfaz de usuario de Scratch. Haciendo un clic sobre "compartir" los proyectos suben a la página Web de Scratch para ser compartidos. Otras personas apoyarán, criticarán y construirán sobre los proyectos de otros, el objetivo es que finalmente resulte una experiencia de aprendizaje interactiva y enriquecedora para todos (Kim et al., 2012). El uso de este programa puede servir para contribuir al desarrollo de algunas habilidades de orden superior y directamente relacionada con la adquisición de competencias clave de los estudiantes. Entre las habilidades que Scratch puede contribuir a desarrollar estarían (Scaffidi \& Chambers, 2012):

- \$Q QUVY. La capacidad para distinguir y separar las partes de un todo hasta llegar a conocer sus principios o elementos.

- 6tQMAMV. Capacidad para llegar a la composición de un todo a partir del conocimiento y reunión de sus partes.

- Conceptualización: La capacidad de abstraer los rasgos que son necesarios y suficientes para describir una situación, un fenómeno o un problema.

- 0 DQHALGHIQRLP DFIyQ. Capacidad para visualizar y ubicar los datos y la información necesarios para la mejor comprensión de un fenómeno o situación dada; discernir la pertinencia de datos e informaciones disponibles y encontrar tendencias o relaciones entre conjuntos desordenados de datos o informaciones.

- 3 HQMP IHQMRIMWPP IFR: La capacidad para visualizar como un sistema los elementos constitutivos de una situación o fenómenos, así como la habilidad de visualizar los sistemas como totalidades que forman parte de totalidades mayores y que pueden ser descompuestos en totalidades menores. Operativamente implica las capacidades de análisis y síntesis, pero agrega el carácter dinámico y se centra en el estudio de las interacciones.

- 3 HQMP IHQWRIFUURR: Capacidad de pensar por cuenta propia, analizando y evaluando la consistencia de las propias ideas, de lo que se lee, de lo que se escucha, de lo que se observa.

- , QYHWJDFIyQ. La capacidad para plantear interrogantes claros con respecto a una situación o fenómeno dado; de proponer hipótesis precisas y modelos conceptuales de lo que se estudia; de producir o recopilar datos e información con el propósito de verificar el modelo conceptual y las hipótesis; se examina el peso y la validez de la información y el grado con el que se refutan las hipótesis o los modelos conceptuales y, por último, formular teorías, leyes o conceptos acerca del fenómeno en estudio.

- 0 HMFRJQIFIy La capacidad de reflexionar sobre los pensamientos propios, incluye la planeación antes de una tarea, el monitoreo durante una tarea y la autoevaluación al terminarla.

El uso de una herramienta gratuita e intuitiva como Scratch puede permitir que el alumno desarrolle de forma interdisciplinar diferentes contenidos de distintas asignaturas y trabaje simultáneamente diversas competencias básicas desde el razonamiento crítico. En cada proyecto es necesario coordinar tiempo, interacciones entre personajes, ubicación y direccionalidad, entre otros muchos aspectos. Esto fomenta en el alumnado la destreza de afrontar situaciones problemáticas, buscar la manera de solucionarlas, el uso de la técnica ensayo-error y la crítica constructiva; conceptos que forman parte del pensamiento sistemático. Los resultados más positivos de la experiencia se han concentrado en la posibilidad de trabajar en grupo interactuar y ayudar a los compañeros en la resolución de dudas de la tarea de programación. Los aspectos 
mejorables es que, en ocasiones, es un procedimiento muy repetitivo, no se pueden aplicar las experiencias a diferentes situaciones ni emplear dispositivos táctiles porque dificulta la tarea de programación. Los estudiantes perciben un aumento de su motivación, una mayor interacción en el proceso de enseñanzaaprendizaje y pone en práctica conceptos teóricos aprendidos en el aula.

Asimismo, la creación de estos proyectos fomenta el trabajo colaborativo, la coevaluación, la imaginación del alumnado y controlar, desde una mayor autonomía, su proceso y su propio aprendizaje. Existen también una serie de aspectos mejorables que hay que ir mejorando como su aplicabilidad a situaciones reales o su empleo con dispositivos digitales como Tablet y smartphone de una manera amigable. El tiempo dedicado para la preparación de estas actividades es también otro elemento que hay que repensar y medir conforme a los resultados obtenidos.

\section{Agradecimientos}

Este trabajo se ha elaborado en el marco del Proyecto I+D+I titulado: "Gamificación y aprendizaje ubicuo en Educación Primaria. Elaboración de un mapa de competencias y recursos docentes, discentes y parentales (GAUBI)" (RTI2018-099764-B-100) (MICINN /FEDER), financiado por el Fondo Europeo de Desarrollo Regional (FEDER) y Ministerio de Ciencia, Innovación y Universidades de España.

\section{Referencias}

BBC (2014). \&RP SXWHUFRGQJ IWXJKWWQ( WRQDQSUP DUपVFKRRQRKhttp://www.bbc.com/news/education25648769 $>$ [Consulta: 18 de marzo de 2020]

BURKE, R. J., \& MATTIS, M. C. (2007). : RP HQDQGO IQRUUMHIQ6FHPFHIZHFQRQRJ प( QJ IQHUQJDQG 0 DUKHP DUFV. Elgaronline. <http://www.elgaronline.com/view/9781845428884.00026.xml $>$ [Consulta: 18 de marzo de 2020]

"What most schools don't teach". $<R X 7 X E H<$ https://www.youtube.com/watch?v=nKIu9yen5n $>$ [Consulta: 22 de diciembre de 2019]

DETERDING, S. (2012). 7KH* DP HXO\&DMRRP $\$$ \$ZRLMKRSDD* DP H. Learning \& Society 8.0. DRUIN, A. (1998). 7KH' HMJQRILKKGUHQIIU7 FKQRRJ।. Morgan Kaufmann Publishers.

FESSAKIS, G., GOULI, E., \& MAVROUDI, E. (2013). "Problem solving by 5-6 years old kindergarten children in a computer programming environment: a case study". \&RP SXWWW L( GXFDMRQU1, 87-97.

GOLD, V. (2011). Students with disabilities, supporting literacy. 6FWWFKHE ' LWFXWRQV. $<$ http://scratched.media.mit.edu/discussions/researching-scratch/students-disabilities-supporting-literacy> [Consulta: 17 de marzo 2020]

KIM, H., CHOI, H., HAN, J., \& SO, H. (2012). "Enhancing teachers' ICT capacity for the 21 st century learning environment: three cases of teacher education in Korea". \$XWDDDMDQ-RXLQDORI ( GXFDURQDO

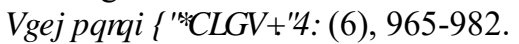

KORDAKI, M. (2012). "Diverse categories of programming learning activities could be performed within



LAMB, A., \& JOHNSON, L. (2011). "Scratch: computer programming for 21st century learners". 7HDFKH / IEUUDQWI (4), 64-68.

LTS. (2010). Curriculum for Excellence. Technologies: experiences and outcomes (Govt. Rep.). Learning and Teaching Scotland (LTS). <http://www.ltscotland.org.uk/curriculumforexcellence/technologies/> [Consulta: 14 de junio 2020]

MATLIN, M. W. (1993). 7KHSW FKR囚J IRIZRP HQ. New York, NY: Harcort, Brace, Jovanovich.

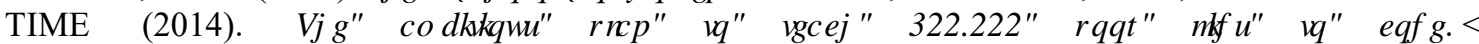
http://time.com/2901198/computer-code-van-jones-prince-yeswecode/ $>$ [Consulta: 23 de marzo de 2020]

SÁEZ LÓPEZ, J.M., ROMÁN, M., \& VÁZQUEZ-CANO, E. (2016). "Visual programming languages integrated across the curriculum in elementary schools: A two year case study using "Scratch" in five

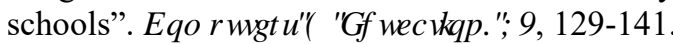

SCAFFIDIi, C., \& CHAMBERS, C. (2012). "Skill progression demonstrated by users in the Scratch animation environment". , QUHQDURQDO- RXLQDORID+ XP DQ \&RP SXWU, QUHDFURQW (6), 383-398.

VÁZQUEZ-CANO, E., \& FERRER DELGADO, D. (2015). "La creación de videojuegos con Scratch en Educación Secundaria". \&RP P XQIFDURQ13 DSHWW, 63-73. 
WARTELLA, E. A., \& JENNINGS, N. (2000). "Children and computers: New technology-old concerns". \&KIQUHQDQCFFRP SXWUUWFKQRQRJ W

WILSON, A., HAINEY, T., \& CONOLLY, T.M. (2013). "Using Scratch with primary school children: an evaluation of games constructed to gauge understanding of programming concepts". , QULQDURQDO RXLDDO $R \square^{*} D P$ H\%DHCVV HDQQQJ

ZICHERMMAN, G., \& CUNNINGHAM, C. (2011). * DP IIFDURQEI I' HMUQ. Sebastopol, CA: O'R 